Ann. Zootech., Ig69, 18 (2), I85-I96.

\title{
IMPORTANCE, COMPOSITION ET SIGNIFICATION DES DIFFÉRENTES FRACTIONS DE LAIT OBTENUES SUCCESSIVEMENT AU COURS DE LA TRAITE MÉANIQUE DES BREBIS
}

\author{
J. LABUSSIÈRE \\ Avec la collaboration technique de J.-F. Combaud, \\ P. Petrequin, R. Tessonnière et R. Govget \\ Laboratoire de Physiologie de la Lactation, \\ Centre national de Recherches zootechniques, 78 - Jouy-en-Josas \\ Institut national de la Recherche agronomique
}

\section{SOMMAIRE}

Certaines brebis Préalpes $d u S u d$ ne présentent pas de réflexe neuro-endocrinien d'éjection au cours de la traite mécanique. Cette absence est due à une rétention importante du lait dans les parties supérieures de la glande.

La suppression des opérations d'égonttage chez ces animaux risquerait de réduire sensiblement la sécrétion du lait et la quantité de matières grasses extraites. En effet, le taux butyreux s'accroît d'une façon importante au cours de la traite. Il est environ trois fois plus fort dans le lait résiduel que dans le lait citernal. Cet accroissement est plus important le matin que le soir et il est d'autant plus net que les brebis présentent un bon réflexe d'éjection. Le taux azoté est peu modifié d'un échantillon à l'autre, néanmoins il est légèrement plus faible dans la partie résiduelle. Dans le cas où la mamelle est vidée complètement de son lait par l'ocytocine, on constate à la traite suivante une réduction significative du lait citernal, et une diminution systématique du taux butyreux dans toutes les fractions.

Des hypothèses sont proposées pour expliquer ce phénomène.

\section{INTRODUCTION}

L'étude des changements de composition du lait entre le début et la fin de la traite (et particulièrement celle du taux butyreux) a été réalisée par de nombreux chercheurs sur de nombreuses espèces (voir l'article de JoHanson, Korkman et NEL,SON, I952, et ceux plus récents de KulikOV, I959 ; PAviov et BrESLAW, I959 ; Poryadkova, I964; Shwabe, Medvedev et Kalantar, ig65; Rotemberg et BARANOWSKI, I965). 
Toutefois, dans la quasi-totalité des cas, les échantillons successifs furent prélevés selon une séquence constante de temps ou de volume ne pouvant tenir compte du déroulement physiologique de l'évacuation du lait, comme il est possible de le faire chez les Ovins.

En effet, les travaux effectués par LABussiÈre et MarTinet (I964) et LABusSI亡̀rRE (I966) ont montré que la traite des brebis à la machine permet de distinguer deux types d'animaux (fig. I) :

a) ceux fournissant leur lait en deux émissions successives bien distinctes, ce qui correspond à l'obtention mécanique du lait citernal puis du lait alvéolaire chassé des acini sous l'effet du réflexe d'éjection (LABussiz̀re, MARTinet et Denamur, Ig69) ;

b) ceux ne présentant que la première émission, la seconde étant absente pour des raisons qui nous sont encore inconnues (inhibition des processus neuro-endocriniens, causes morphologiques, etc.).

Il est aisé de collecter et d'analyser séparément le lait de ces deux émissions, ainsi que celui obtenu par les différentes manipulations qui complètent les opérations de traite : égouttage-machine, égouttage manuel et lait résiduel.

C'est ce que nous avons fait au cours de l'expérience que nous nous proposons de rapporter. Cet essai nous a paru justifié puisqu'il existe peu de références bibliographiques consacrées à cette question chez la Brebis. Il faut citer les articles de Semjam (i962), Ricordeau, Martinet et Denamur (ig63), Aminov (ig66), en signalant toutefois que ce dernier a effectué ses analyses sur des portions successives obtenues par cathétérisation du trayon, et non dans les conditions de la traite proprement dite comme c'est le cas pour notre travail.

\section{MATÉRIEL, ETT TECHNIQUES}

\section{A. - Conduite générale du troupeau}

L'agneau tète librement pendant les trois premiers jours qui suivent la mise bas. Il est ensuite séparé de sa mère qui passe alors sans transition à la traite mécanique.

Cette opération, qui a lieu deux fois par jour à 6 heures et $16 \mathrm{~h}{ }_{15}$, est effectuée sur un "carroussel "à trente-deux places, à l'aide d'une machine Alfa-Laval dont les caractéristiques principales sont les suivantes :

Vide : $33 \mathrm{~cm}$ de mercure.

Vitesse de pulsation : I $80 /$ minute.

Rapport succion/massage $=\mathrm{r} / \mathrm{r}$.

Les gobelets pesant environ $240 \mathrm{~g}$ sont posés sans préparation préalable de la mamelle et sont enlevés après qu'un égouttage-machine ait été pratiqué. Enfin, une extraction plus complète du lait est assurée par un égouttage manuel ou " repasse ".

Les 57 brebis qui ont servi pour cet essai se répartissent de la façon suivante :

- 32 à deux émissions, dont I 3 en première lactation, i I en seconde et 8 en troisième ou plus.

- 25 à une émission, dont i I en première lactation, 4 en seconde et ro en troisième ou plus.

\section{B. - Technique des contrôles}

Les différentes fractions obtenues au cours de la traite ont déjà été décrites dans une étude précédente. Il nous paraît néanmoins utile de rappeler les principales définitions, certaines étant schématisées à la figure $\mathbf{I}$. 
Lait machine (en $\mathrm{ml}$ ) : volume de lait obtenu par la machine sans intervention du trayeur.

Volume du $7^{\mathrm{er}}$ pic $\left(\mathrm{V}_{1}\right.$ en $\left.\mathrm{ml}\right)$ : volume de lait recueilli avant l'apparition de la $2^{\mathrm{e}}$ émission. Il représente le lait citernal.

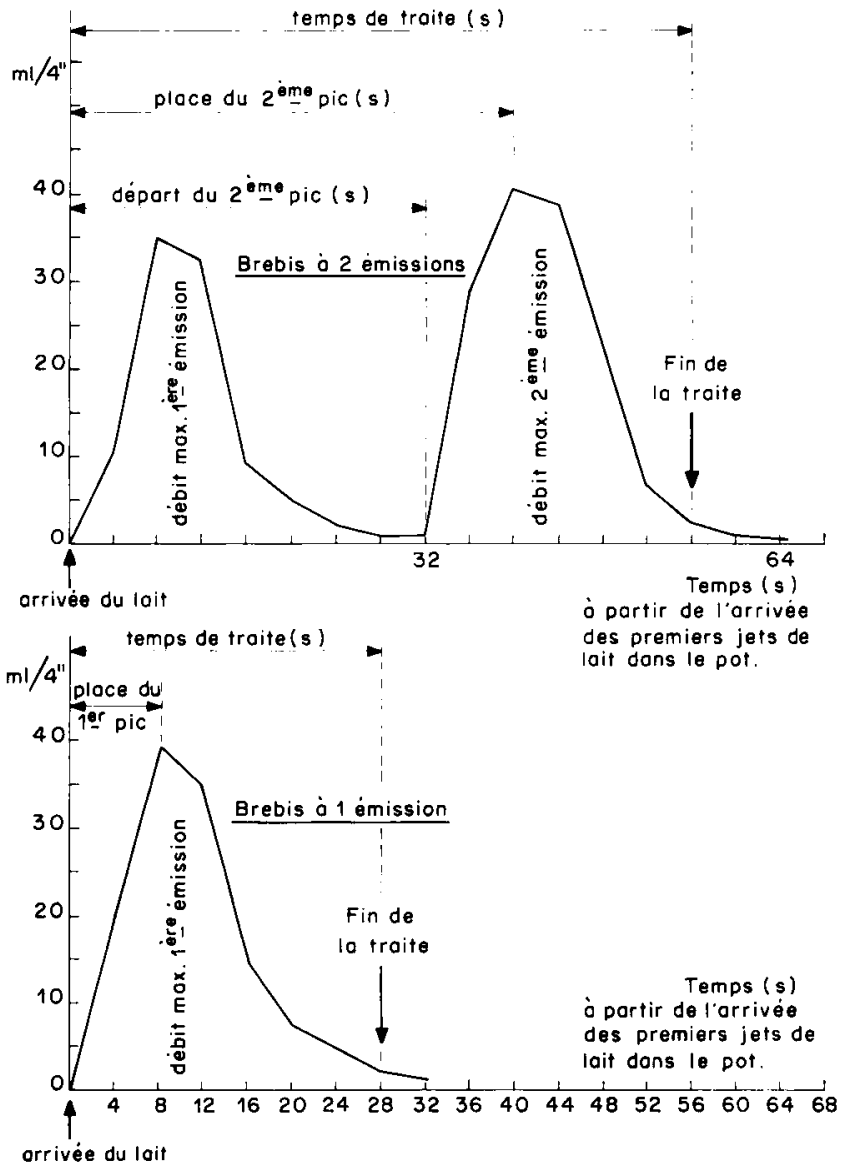

FIG. I. - Les deux types de descente du lait au cours de la traite mécanique des brebis

Volume du $2^{\mathrm{e}}$ pic $\left(\mathrm{V}_{2}\right.$ en $\mathrm{ml}$ ) : lait machine moins volume du I er pic. Il représente le lait alvéolaire (chassé des acini par l'ocytocine) obtenu sans intervention du trayeur.

Égouttage machine (en $\mathrm{ml}$ ) : volume de lait obtenu à la machine lors du massage par le trayeur.

Égouttage manuel (ou repasse) (en $\mathrm{ml}$ ) : volume de lait obtenu lors de la traite à la main, après l'enlèvement des gobelets.

Ces fractions successives sont recueillies dans des pots séparés grâce à un dispositif de robinets à plusieurs voies. Le lait de "repasse " est récolté sans difficultés particulières.

Une administration intra-jugulaire de 2 UI d'ocytocine (Syntocinon Sandoz) est alors effectuée, afin d'extraire le lait résiduel qui est obtenu par un deuxième égouttage manuel.

Les différents échantillons sont mesurés volumétriquement et analysés pour leur teneur en matières grasses (méthode de Gerber) et en matières azotées (méthode au noir amido) (1). Leur importance absolue et relative est représentative pour chaque animal des conditions normales d'éjection du lait, puisqu'aucune injection d'ocytocine n'a été pratiquée au cours des traites précédentes. Ces données constituent les résultats témoins. Afin de rechercher les mécanismes responsables de l'écoulement du lait dans la citerne entre les traites, les mêmes contrôles sont répétés à la traite suivante.

(1) Ces analyses ont été effectuées par la Station expérimentale laitière de Poligny (Jura). 
Dans ce dernier cas, en effet, la répartition des produits de sécrétion peut être influencée par la vacuité de la glande mammaire produite par l'ocytocine. Ces données constituent les résultats expérimentaux. L'essai est organisé suivant le schéma indiqué au tableau I. Il a lieu au cours de la sep-

TABLEAU I

Schéma expérimental

\begin{tabular}{|c|c|c|c|c|}
\hline & \multicolumn{2}{|c|}{ Premier groupe } & \multicolumn{2}{|c|}{ Deuxième groupe } \\
\hline & $\begin{array}{l}\text { Caractéristiques } \\
\text { de la traite }\end{array}$ & Contrôle & $\begin{array}{l}\text { Caractéristiques } \\
\text { de la traite }\end{array}$ & Contrôle \\
\hline $\begin{array}{l}\text { Dimanche soir } \\
\text { Lundi matin } \\
\text { Lundi soir } \\
\text { Mardi matin } \\
\text { Mardi soir } \\
\text { Mercredi matin } \\
\text { Mercredi soir } \\
\text { Jeudi matin } \\
\text { Jeudi soir }\end{array}$ & $\begin{array}{c}\text { normale } \\
\text { finition à l'ocytocine } \\
\text { finition à l'ocytocine } \\
\text { normale } \\
\text { normale } \\
\text { normale } \\
\text { finition à l'ocytocine } \\
\text { finition à l'ocytocine } \\
\text { normale }\end{array}$ & $\begin{array}{c}\text { néant } \\
\text { témoin } \\
\text { expérimental } \\
\text { néant } \\
\text { néant } \\
\text { néant } \\
\text { témoin } \\
\text { expérimental } \\
\text { néant }\end{array}$ & \begin{tabular}{|} 
normale \\
normale \\
finition à l'ocytocine \\
finition à l'ocytocine \\
normale \\
normale \\
normale \\
finition à l'ocytocine \\
finition à l'ocytocine
\end{tabular} & $\begin{array}{c}\text { néant } \\
\text { néant } \\
\text { témoin } \\
\text { expérimental } \\
\text { néant } \\
\text { néant } \\
\text { néant } \\
\text { témoin } \\
\text { expérimental }\end{array}$ \\
\hline
\end{tabular}

tième semaine qui suit la mise-bas. Au fur et à mesure qu'elles arrivent à ce stade, les brebis sont affectées alternativement au premier, puis au deuxième groupe. En effet, dans le premier groupe les contrôles témoins et expérimentaux du matin interviennent respectivement en début et en fin de semaine. Ainsi le contrôle expérimental pourrait être systématiquement pénalisé par un léger décalage dans le stade de lactation. L'introduction d'un deuxième groupe permet de corriger cet: éventuel effet parasite.

\section{RÉSUITATS}

\section{A. - I:mportance et composition des fractions recueillies dans les conditions normales de traite (contrôle témoin)}

Les résultats sont présentés au tableau 2 . Ils appellent les remarques suivantes :

\section{Production laitière.}

a) Chez les brebis à un pic, les rolumes des égouttages machine et manuel et celui de la fraction résiduelle sont plus importants que chez les brebis à deux pics, alors que la première partie citernale ne diffère pas significativement d'un type d'animal à l'autre.

Il semble donc que l'absence de la deuxième émission soit essentiellement due à une rétention du lait dans les parties supérieures de la glande.

Cette rétention est considérable puisque, par exemple, le lait résiduel du matin contient environ $37 \mathrm{p}$. roo des matières grasses sécrétées $\left({ }^{1}\right)$ alors que la machine seule (sans participation du trayeur) n'en extrait que 25 p. Ioo.

(1) Johansson, Korkman et Nelson (1952) trouvent environ 32 p. 100 chez la Vache, et Ricordeau Martinet et Denaulr (1963) des résultats comparables chez la Brebis. 
COMPOSITION DU IAIT E'T 'TRAITE MÉCANIQUE DES BREBIS

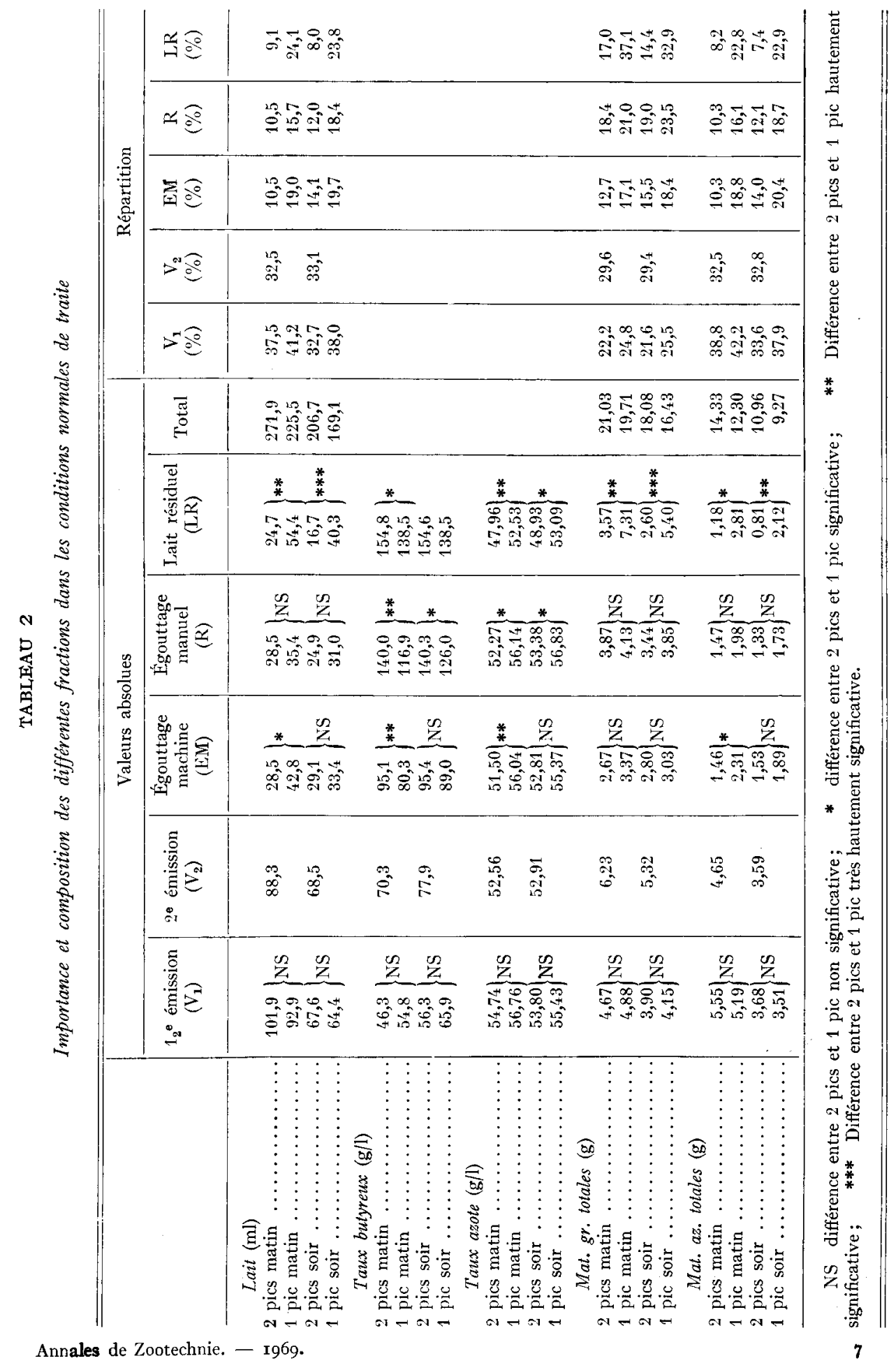


b) Chez les brebis à deux émissions, il existe une corrélation positive hautement significative entre :

- le lait total et le volume du deuxième pic $(r=+0,63$ et $r=+0,56$, respectivement aux traites du soir et du matin) ;

- le lait total et la quantité de lait résiduel $(r=+0,49$ et $r=+0,55$, respectivement aux traites du soir et du matin).

L'interrelation entre ces trois facteurs explique probablement pourquoi nous n'avons pas trouvé de liaison entre le volume du deuxième pic et celui du lait résiduel $(r=0$, OI $)$.

Toutefois, lorsque le lait total est maintenu constant, on obtient une corrélation partielle négative hautement significative entre ces deux critères $(r=-0,473$ et $r=-0,506)$; la deuxième émission étant l'extériorisation dans le pot à traire du réflexe d'éjection du lait (LABUssière, Martinet et Denamur, I969), il est en effet logique d'admettre que plus ce dernier est intense, plus le lait résiduel est faible.

\section{Taux butyrenx.}

Comme l'ont déjà remarqué de nombreux chercheurs (voir les revues bibliographiques citées dans 1'introduction), on constate que le taux butyreux augmente

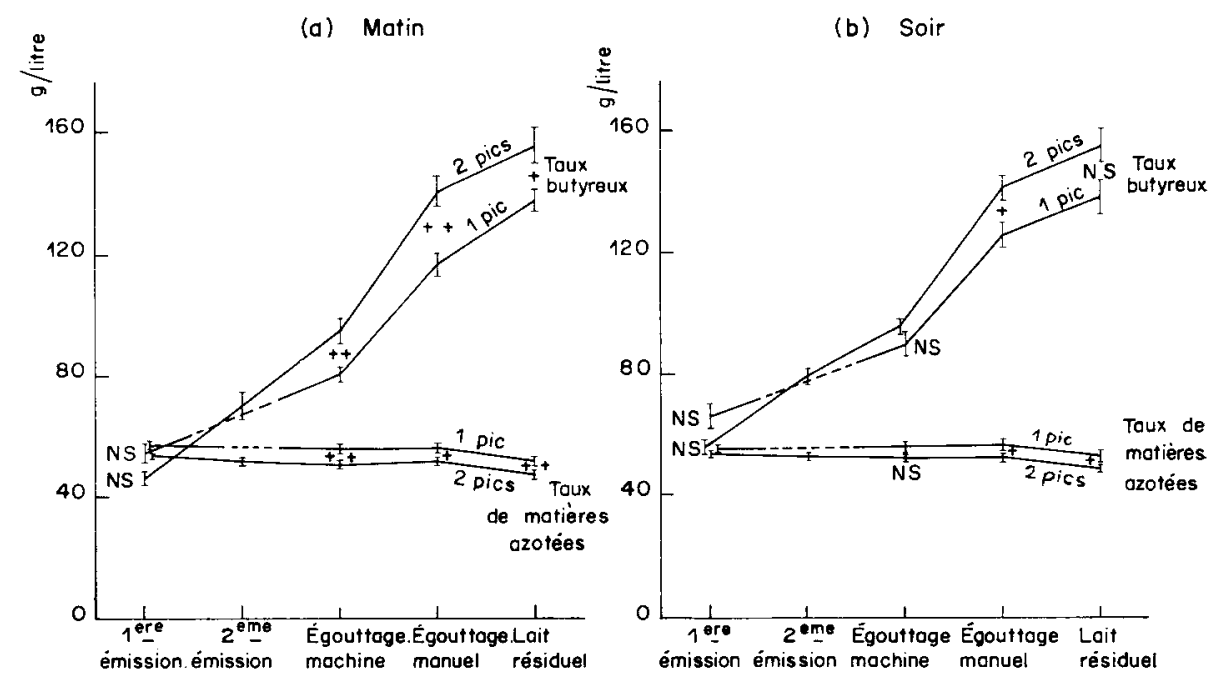

FIG. 2. - Évolution du taux butyreux et du taux de matières azotées au cours de la traite

+ différence entre $I$ pic et 2 pics significative

++ différence entre $I$ pic et 2 pics hautement significative

NS différence entre 1 pic et 2 pics non significative

très sensiblement au cours de la traite (voir aussi fig. 2). Cet accroissement semble d'autant plus important que l'aptitude à la traite est grande puisque :

a) Il est plus marqué chez les brebis présentant un deuxième pic, c'est-à-dire un réflexe d'éjection du lait (LABussière, Martiner et Denamur, rg69);

b) Il est d'autant plus fort que le lait résiduel est faible $\left(r_{1}=-0,238\right.$; 
$r_{2}=-0,489^{* *} ; r_{3}=-0,379^{* *} ; r_{4}=-0,475^{* *}$ suivant le type de contrôle effectué) (1).

Il importe également de faire deux remarques concernant la traite du matin :

a) L'écart entre le taux butyreux de la première fraction et celui de la dernière est plus élevé qu'à la traite du soir (tabl. 3) ;

\section{TABLEAU 3}

Augmentation du taux butyreux entre la première et la dernière fraction

\begin{tabular}{|c|c|c|c|c|c|}
\hline & \multirow[b]{2}{*}{ Type de brebis } & \multicolumn{2}{|c|}{ Matin } & \multicolumn{2}{|c|}{ Soir } \\
\hline & & Moyenne & $\begin{array}{c}\begin{array}{c}\text { Erreur } \\
\text { standard }\end{array} \\
\sqrt{\mathrm{S}^{2} / n}\end{array}$ & Moyenne & $\begin{array}{l}\text { Erreur } \\
\text { standard } \\
\sqrt{\mathrm{S}^{2} / n}\end{array}$ \\
\hline $\begin{array}{l}\text { Écart entre première } \\
\text { fraction et dernière } \\
\text { fraction en } \mathrm{g} / 1000\end{array}$ & $\begin{array}{l}2 \text { pics } \\
1 \text { pic }\end{array}$ & $\begin{array}{r}106 \\
81\end{array}$ & $\begin{array}{l}4,8 \\
5,3\end{array}$ & $\begin{array}{l}89 \\
66\end{array}$ & $\begin{array}{l}5,7 \\
5,5\end{array}$ \\
\hline
\end{tabular}

b) Les taux butyreux des laits d'égouttage (machine et manuel) ainsi que celui du lait résiduel sont semblables à leurs homologues de la traite du soir (tabl. 2). Ceci n'est valable que pour les brebis à deux émissions.

\section{Taux de matières azotées.}

Le taux de matières azotées est relativement stable au fur et à mesure du déroulement de la traite (fig. 2); toutefois, celui de la première émission est légèrement plus élevé que celui de la dernière fraction extraite à l'ocytocine. Ces résultats confirment ceux obtenus chez la Vache par Johansson, Korkman et Nelison (I952), Kulikov (I959), Poryadkova (I964), Lewis et Davies (I965), Shwabe, MEDVEDEV et Kalantar (1965).

Ils sont valables pour les deux types d'animaux, mais les brebis à un pic ont toujours un lait plus riche que celui des brebis à deux pics.

\section{B. - Importance et composition des différentes fractions recueillies au cours des traites expérimentales \\ (injection d'ocytocine à la fin de la traite précédente)}

Quand on extrait le lait résiduel restant dans la mamelle après 1'égouttage manuel, on constate à la traite suivante de nombreuses perturbations.

(1) $r_{1}=$ contrôle témoin matin.

$r_{2}=$ contrôle expérimental matin.

$r_{3}=$ contrôle témoin soir.

$r_{4}=$ contrôle expérimental soir.

Le signe ** correspond à une corrélation hautement significative. 
I. Pour la production laitière.

- Le lait citernal (premier pic) est réduit d'une façon importante, surtout chez les brebis à une émission où l'effet est hautement significatif (fig. 3). Ce phénomène est également observé chez la Vache par plusieurs chercheurs (voir l'ouvrage de ZAKS, I962).
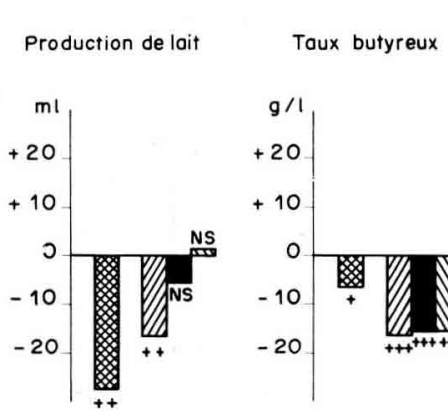

\section{Brebis d 1 pic}
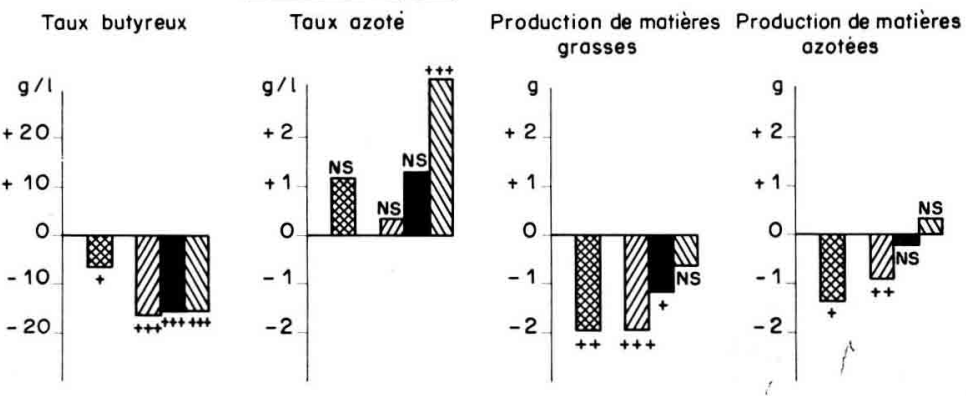

Brebis à 2 pics

Production de lait Toux butyreux

Toux ozoté

Production de motières

Production de matieres
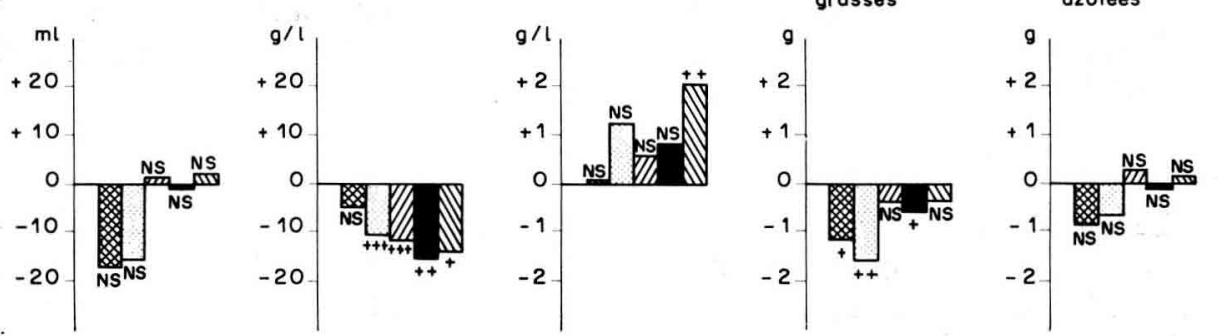

FIg. 3 - Analyse des différentes tractions d'une traite expérimentale (soir)

Les résullats expriment l'écart (positif ou négatif) par rapport à une traile témoin

Cet écart peut être, NS non significatif

* significatif ( 5 p. Ioo)

** hautement significatif ( 1 p. Ioo)

*** très hautement significatif $(0,1$ p. I00)

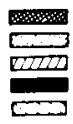

fraction citernale (I re émission)

fraction alvéolaire ( $2^{e}$ émission)

égouttage machine

égouttage manuel

lait résiduel

- Les autres fractions sont d'autant moins affectées qu'elles sont recueillies plus tardivement au cours du déroulement de la traite, le lait résiduel restant d'ailleurs identique à celui obtenu dans les conditions " normales ".

2. Pour les matières grasses.

Le taux butyreux est systématiquement plus faible dans toutes les fractions récoltées (fig. 3). 
Ce résultat, qui dans certains cas est très hautement significatif, ne peut s'expliquer par un effet de dilution, puisque les volumes de lait correspondants sont également plus faibles. Il est possible que cette diminution soit le " contrecoup " de la reconstitution d'un " stock lipidique alvéolaire permanent " qui avait été extrait précédemment, lors de l'obtention du lait résiduel, mais bien d'autres hypothèses peuvent être formulées à ce sujet et il nous est difficile d'apporter des arguments en faveur de l'une d'entre elles.

\section{Pour les matières azotées.}

Contrairement aux taux butyreux, les taux azotés de tous les prélèvements sont légèrement plus élevés que ceux observés lors du contrôle témoin (fig. 3).

Il est intéressant de remarquer que cette inversion des résultats entre ces deux critères avait également été observée en ce qui concerne l'évolution de la composition du lait au cours de la traite, puisqu'alors (fig. 2) :

le taux butyreux augmente,

le taux azoté diminue.

Toutes les modifications que nous venons d'évoquer (lait, matières grasses, azote) sont plus importantes le soir que le matin et ceci s'explique probablement par le fait que l'intervalle diurne étant plus court que l'intervalle nocturne, la répartition du lait dans la glande demeure encore très affectée par l'injection d'ocytocine (le retour à l'état antérieur étant plus complet dans le deuxième cas).

\section{DISCUSSION ET CONCLUSION}

L'évolution du taux butyreux et du taux azoté que nous constatons chez la Brebis depuis l'extraction des premiers jets de lait jusqu'à celle du lait résiduel, concorde avec celle observée dans cette espèce par SEMJAM (I962), Ricordeau, Martinet et Denamur (I963), Aminov (i966), et chez la Vache par d'autres auteurs.

Il importe toutefois de rappeler que contrairement à de nombreux travaux effectués jusqu'à maintenant, celui-ci a permis d'analyser toutes les fractions de lait émises au cours d'une traite normale. Ces fractions ayant une signification physiologique, ceci nous autorise à émettre quelques hypothèses sur les mécanismes de l'écoulement du lait dans la mamelle entre les traites et pendant la traite.

\section{Entre les traites}

Dans les conditions normales, une partie seulement du lait sécrété s'écoule dans la citerne. On peut en disposer immédiatement lors de la pose des gobelets.

Dans le cas où la glande mammaire a été vidée de tout son lait par l'ocytocine quelques heures auparavant, on constate une diminution sensible de cette fraction citernale, les autres échantillons (deuxième émission, égouttages machine et manuel, lait résiduel) étant beaucoup moins affectés. Le phénomène est d'ailleurs d'autant plus net que l'intervalle horaire séparant cette observation de l'administration de l'ocytocine est court. 
Il semblerait donc qu'il y ait remplissage préférentiel des parties supérieures de la glande au détriment des parties les plus basses.

Doit-on penser que le lait citernal représente le "surplus " de ce qui ne peut être contenu dans les acini? Les travaux que nous poursuivons actuellement au laboratoire ne nous permettent pas de conclure positivement à ce sujet, et 1'on peut penser que des processus physiologiques beaucoup plus complexes entrent en jeu.

\section{Pendant la traite}

L'absence de la deuxième émission au cours de la traite des brebis à un pic peut avoir deux significations :

a) Il y a eu descente prématurée de ce lait dans la citerne, avant la pose des gobelets, et ceci sous l'influence de stimuli conditionnels pouvant provoquer la décharge d'ocytocine (arrivée du trayeur, mise en route des pulsateurs de la machine, etc.).

Ceci ne paraît pas être le cas, car les taux butyreux et azoté ainsi que les volumes de la première fraction recueillie chez les brebis à un et deux pics, ne diffèrent pas significativement.

b) Il y a eu rétention du lait à proximité des cellules sécrétoires pour des raisons physiologiques ou morphologiques qu'il ne nous est pas encore possible de préciser.

Tel semble bien être le cas, puisque l'absence de la deuxième émission s'accompagne de l'augmentation des égouttages machine et manuel et, surtout, du lait résiduel.

Il faudra tenir compte de cette mauvaise évacuation si l'on envisage de supprimer les manipulations qui accompagnent fréquemment la fin de la traite; chez les brebis à un pic qui s'avèrent donc inaptes à la traite mécanique, cette quantité importante de lait, riche en matières grasses, qui serait laissée dans la mamelle, risquerait en effet de :

- freiner le métabolisme des cellules sécrétrices, et ainsi de réduire la production;

- modifier sensiblement la qualité fromagère du lait.

Dans le cas présent, cette rétention partielle contribue probablement à diluer les fractions grasses alvéolaires tout en empêchant leur mobilisation; il est donc normal que l'accroissement du taux butyreux soit moins fort pour les brebis à un pic que pour celles qui sont faciles à traire et également meilleures laitières (brebis à deux pics). Chez ces dernières, en effet, on peut estimer que le lait retenu représenterait principalement une "constante physique " dépendant surtout de la morphologie de la mamelle (effets de capillarité des petits canaux, position des trayons, etc.). On comprend alors pourquoi, chez les brebis à 2 pics le taux butyreux des fractions d'égouttage et du lait résiduel reste relativement constant, quelles que soient 1'heure de la traite et la quantité de lait sécrétée. A ce sujet, il faut remarquer que Johansson, KORKMAN et Neison (1952) trouvent une augmentation de la teneur en matières grasses entre la première et la dernière fraction :

a) plus importante chez les vaches bonnes productrices et à grand débit; ces résultats étant à rapprocher de ceux observés chez les brebis à deux pics ;

b) plus forte le matin que le soir, ce qui est aussi notre cas.

A l'examen de la figure 2 on peut admettre que ce dernier phénomène est surtout la conséquence d'un taux butyreux plus faible le matin que le soir dans la partie 
citernale. Il pourrait s'agir, là encore, d'un cas de dilution, le "surplus " citernal étant plus important après un long intervalle de traite.

Signalons enfin que chez les brebis à deux pics, il existe un certain "gradient " dans l'efficacité du réflexe d'éjection. Par un calcul de corrélation partielle éliminant le facteur "niveau de production ", il ressort en effet que le lait résiduel est d'autant plus élevé que la deuxième émission est plus petite $(r=-0,473$ et $r=-0,506$, respectivement aux traites du soir et du matin). Des études ultérieures mériteraient d'être entreprises pour expliquer ce phénomène.

Reçu pour publication en mai 1969 .

\section{REIMERCIEMENTS}

Nous tenons à remercier le personnel de la Ferme expérimentale de Brouessy dont l'aide nous a été précieuse pour la réalisation de ce travail.

\section{SUMMARY}

AMOUNT, COMPOSITION AND SIGNIFICANCE OF VARIOUS MILK FRACTIONS

SUCCESSIVELY OBTAINED DURING THE MECHANICAL MILKING OF EWES

Various fractions of the milk of 57 Préalpes $d u$ Sud ewes, emitted under ordinary mechanical milking conditions, were collected, measured, and analyzed separately. These fractions have physiological significance ; that is, they correspond to the successive extraction of cistern milk, alveolar milk driven from the acini by the neuro-endocrine ejection reflex, machine and manualstripped milk, and residual milk obtained after an intra-jugular injection of 2 IU of oxytocin.

I. 25 of the 57 ewes studied did not have the second emission characteristic of the neuroendocrine ejection reflex (one-peak ewes). This absence represents a large milk retention in the upper parts of the udder since the volumes of the last three fractions (stripping and residual milk) are almost twice greater in those animals which thus give cistern milk in the machine only (without the interference of a milker) (table $\mathrm{I}$ ).

2. The fat content increases significantly during milking. It is about three times higher in residual milk than in the first jets (fig. 2 and table 11 ). This increase is greater in the morning than in the evening, and more evident when the ewes have a good ejection reflex.

3. The nitrogen content is about the same in all fractions. However, it is a little lower in residual milk (fig. 2 and table $I I$ ).

4. It must be emphasized that total suppression of stripping in one-peak ewes may perceptibly reduce available milk secretion and fat content to $25 \mathrm{p}$. Ioo.

5. When the udder is completely emptied by the administration of oxytocin, there is a significant reduction of cistern milk at the next milking, and a systematic decrease of fat content in all samples taken. Some hypotheses are proposed to explain this phenomenon.

\section{RÉFÉRENCES BIBLIOGRAPHIQUES}

Aminov S. A., ig66. Fat distribution in successives portions of one milking in sheep and goat. Neiro-gormonal'naya-regulijatsuija laktatsii, I39-145. Izdatel'stvo "Nauka ", Moscou. (Dairy Sci. Abstr., I967, page 284$)$.

Johansson I., Korkman N., Nelson N. J., I952. Studies on udder evacuation in dairy cows. I. The rise in fat percentage during milking. Acta Agr. Scand., 2, 43-8 I .

Kulikov L. V., 1959. Changes in the composition of cows'milk in individual milk fractions. Dokl. MoskSel's kokhoz. Akad. Timiryazeva., 45, I $19-124$. 
Labussilire J., Martinet J., i 964. Description de deux appareils permettant le contrôle automatique des débits de lait au cours de la traite à la machine. Premiers résultats obtenus chez la Brebis. Ann. Zootech., 13, I $99-2$ I 2

LABUSSIÉRE J., I 966. Relations entre le niveau de production laitière des brebis et leur aptitude à la traite. XVII' Congrès Int. Laiterie, Munich, Section Al., 43-5I.

Labussikie J., Martinet J., Denamur R., i 969 . The influence of the nilk ejection reflex on the flow rate during the milking of ewes. J.Dairy Sci. (in press).

Lewis J., Davies J., I965. Observations on milk quality with particular reference to foremilk and strippings. J. Soc. Dairy Technol., 18, 2 8-223.

Pavlov G. N., Breslaw I. S., 1959. The physiology of milk-fat secretion. Int. Dairy Congr., 1,8 -I 4.

Poryadkova K. A., 1964. Fat and S. N. F. content of the citemal and alveolar fractions of milk. Trudy kishinev. Sel'khoz Inst., 35, 56-66.

Ricordeau G., Martinet J., Denamur R., i 963 . Traite à la machine des brebis Préalpes du Sud. Importance des différentes opérations de la traite. Ann. Zoolech., 12, 203-225.

Rotemberg S., Baranowski S., 1965. Fat and cholesterol contents of milk at the beginning and end of milking. Acla Physiol. Pol., 16, 465-473.

SEMJAy S., 1962 . The distribution of fat in individual portions of normal and residual milk. Sborn Vysokej-Skoly pol'nohosp, Niira, Prevadzkovo-Ekon-Fak. 6, i 87-209. (Dairy Sci. Abstr., 1963, 25, page 484).

Suware A. K., Medvedev I. K., Kalantar I. L., ig65. Composition and secretion of milk fat and protein at different stages of synthesis. Trudy vses. uaucauo-issled. Inst. Fiziol. Biokhim. Sel'khoz Zhivotn 2, 281-294. (Dairy Sci. Abstr., i 967,29 , page 97).

Zaks M. G., I962. The motor apparatus of the mammary gland (Oliver and Boyd, Edinburgh and London) edited by A. T. CowIE. 